\title{
Development and Experimental Validation of a Combined FBG Force and OCT Distance Sensing Needle for Robot-Assisted Retinal Vein Cannulation
}

\author{
J. Smits ${ }^{1}$, M. Ourak ${ }^{1}$, A. Gijbels ${ }^{1}$, L. Esteveny ${ }^{1}$, G. Borghesan ${ }^{1}$, L. Schoevaerdts ${ }^{1}$, K. Willekens ${ }^{2}$, \\ P. Stalmans ${ }^{2}$, E. Lankenau ${ }^{3}$, H. Schulz-Hildebrandt ${ }^{4}$, G. Hüttmann ${ }^{4}$, D. Reynaerts ${ }^{1}$, E.B. Vander Poorten ${ }^{1}$
}

\begin{abstract}
Retinal Vein Occlusion is a common retinal vascular disorder which can cause severe loss of vision. Retinal vein cannulation followed by injection of an anti-coagulant into the affected vein is a promising treatment. However, given the scale and fragility of the surgical workfield, this procedure is considered too high-risk to perform manually. A first successful robot-assisted procedure has been demonstrated. Even though successful, the procedure remains extremely challenging. This paper aims at providing a solution for the limited perception of instrument-tissue interaction forces as well as depth estimation during retinal vein cannulation. The development of a novel combined force and distance sensing cannulation needle relying on Fiber Bragg grating (FBG) and Optical Coherence Tomography (OCT) A-scan technology is reported. The design, the manufacturing process, the calibration method, and the experimental characterization of the produced sensor are discussed. The functionality of the combined sensing modalities and the real-time distance estimation algorithm are validated respectively on in-vitro and ex-vivo models.
\end{abstract}

\section{INTRODUCTION}

\section{A. Retinal Vein Occlusion and retinal vein cannulation}

Retinal Vein Occlusion (RVO) is a vitreoretinal (VR) eye condition which affects an estimated 16.4 million people worldwide [1]. It is the 2nd most common retinal vascular disorder after diabetic retinal disease. The disease occurs when clots are formed inside a retinal vein (Fig. 1). This causes the patient to slowly lose his/her sight. Today, there is no proven effective treatment clinically available for this disease [2]. A promising treatment is retinal vein cannulation [3]. Through a small incision in the sclera the surgeon would move a needle through the inside of the eye to cannulate and inject a clot-dissolving drug into the affected vein. During this procedure, the surgeon looks through a stereoscopic microscope located directly above the patient's eye. Often, additional wide-angled lenses are placed above the patient's eye lens, to enlarge the field of view of the surgeon. The workspace is illuminated by means of a handheld light

\footnotetext{
${ }^{1}$ Jonas Smits, Mouloud Ourak, Andy Gijbels, Laure Esteveny, Gianni Borghesan, Laurent Schoevaerdts, Dominiek Reynaerts, and Emmanuel Benjamin Vander Poorten are with the KU Leuven - University of Leuven, Department of Mechanical Engineering, 3001 Heverlee, Belgium.

${ }^{2}$ Koen Willekens and Peter Stalmans are with the KU Leuven - University Hospitals Leuven, Department of Ophthalmology, 3000 Leuven, Belgium.

${ }^{3}$ Eva Lankenau is with OptoMedical Technologies GmbH, 23562 Lübeck, Germany.

${ }^{4}$ Hinnerk Schulz-Hildebrandt and Gereon Hüttmann are with the Medical Laser Center Lübeck GmbH, 23562 Lübeck, Germany.

Corresponding Author: jonas.smits@kuleuven.be
}

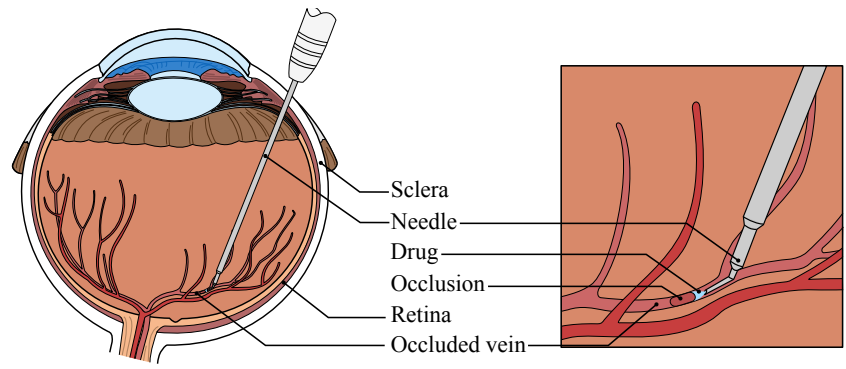

Fig. 1. Retinal vein occlusion and retinal vein cannulation [5].

probe, or a pair of light fibers commonly referred to as "chandeliers". Despite this enhanced visualization of the surgical workspace, physiological hand tremor, limited depth perception, and lack of tactile or force feedback make it highly challenging to perform such a procedure manually. The risk of damaging blood vessels or the retina is extremely high. Due to this, retinal vein cannulation remains a controversial therapy and is not clinically applied today. Recently, the first robot-assisted RVC procedure in a human patient was performed [4].

\section{B. Robotic assistance for retinal surgery}

Since 1989, over a dozen robotic systems for retinal surgery have been reported on in the literature [6]. These systems can be subdivided into three categories: hand-held devices, comanipulation systems and telemanipulation systems. Despite the excellent positioning precision provided by a surgical robot, depth and force perception remain suboptimal. Different sensing technologies have been developed to resolve these issues.

\section{Force sensing for retinal surgery}

Intraocular instrument interaction forces during retinal surgery range from $0.6 \mathrm{mN}$ to $17.5 \mathrm{mN}$ during retinal vein cannulations on porcine ex-vivo and in-vivo eyes [5]. Measurement of instrument-retina interaction forces inside the eye requires forces to be measured distal from the sclera, as it would otherwise not be possible to distinguish scleral contact forces from tip interaction forces [8]. In order to meet size and precision requirements for this application, the usage of FBG sensors is primarily found in the literature. Johns Hopkins University previously reported on a retinal pick and 


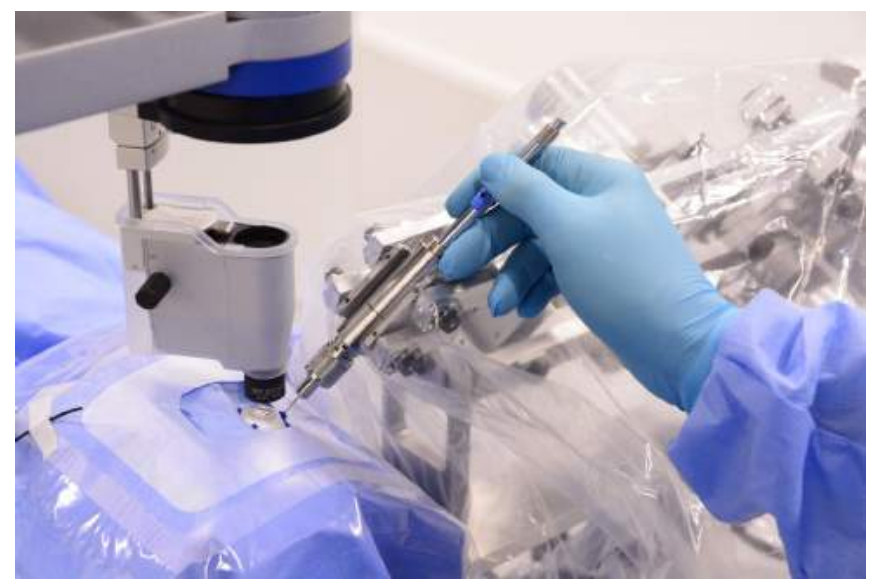

Fig. 2. The developed surgical robotic system at the University of Leuven [7].

an actuated microforceps, both offering 3-DOF force sensing using FBG's [9], [10]. Previous work by both Gonenc et al. as well as Gijbels et al. describe the development of a 2-DOF force sensing cannulation needle using FBG's [11], [12].

\section{Distance sensing for retinal surgery}

An intraoperative Optical Coherence Tomography (iOCT) system provides high-resolution intraoperative imaging of soft tissue anatomy. Typically, 2D or 3D OCT images are generated, referred to as B- and C-scans respectively. An iOCT system shares a large part of its optical path with the surgical microscope. This is convenient as adjustment of zoom lenses affects both imaging modalities equally. Alternatively, OCT data can be acquired via an optical fibre that is incorporated directly inside the surgical instrument. By introducing the surgical instrument in the eye, the complex optical path from the microscope and patient's lens can be effectively by-passed. This allows for more accurate measurements of the retina to be obtained. Only a single ray of laser light can be passed at a time through the laser fiber. The result is a one-dimensional depth scan, also referred to as an A-scan. The development of a single A-scan fiberintegrated retinal pick has been reported on by Balicki et $a l$. [13]. The distance sensing retinal pick was used together with a robotic platform and a custom developed OCT device, and was experimentally validated on synthetic phantoms. Borghesan et al. and Cheon et al. have reported on retinal surface detection algorithms using A-scan data retrieved from ex-vivo enucleated porcine and bovine eyes respectively [14], [15]. To the knowledge of the authors, a single A-scan fiberintegrated cannulation needle has not been developed earlier. A first effort towards such an instrument was made, as the authors reported on a first proof of concept of combined force and distance sensing for vitreo-retinal surgery using FBG and OCT A-scan technology [16]. Here, a single OCT A-scan fibre probe was aligned and fixated to a force sensing cannulation needle. As an early proof of concept, the sensor combination was used together with a previously developed surgical robot to perform retinal vein cannulation on ex-vivo enucleated porcine eyes. Feasibility of combined sensing modalities was shown on dissected porcine eyes, but was however not integrated in a single instrument design. Furthermore, no in-depth analysis or characterisation on the OCT A-scan fibre probe and distance estimation algorithm was performed.

\section{E. Objectives}

This paper aims at providing a first step towards more intraoperative information during retinal vein cannulation. By doing so, we strive to gain better understanding of the cannulation process. Furthermore, the aim is to determine if OCT A-scan distance measurements can be used for further development of robot-assisted safety features such as puncture detection or adaptive admittance control. The development of a novel combined force and distance sensing cannulation needle relying on FBG and OCT Ascan technology is reported. The design and manufacturing process (II), the calibration method and the experimental characterization (III), and the experimental validation (IV) of the produced sensor are discussed. The combined sensing modalities and the functionality of the real-time distance estimation algorithm are experimentally validated on in-vitro and ex-vivo models respectively. The results of this experimental validation will serve as a benchmark for future development of distance estimation algorithms and integration of OCT A-scan technology with a surgical robotic platform.

\section{DEVELOPMENT OF THE SENSOR}

This section describes the development of a combined force and distance sensing cannulation needle based on FBG force and OCT A-scan distance measurements. Firstly, the conceptual design and functionality of the sensor is described. Finally, the detailed design and the assembly process of this novel sensorized instrument are reported. The design of the previously developed 2-DOF force-sensitive needle by Gijbels et al. [12] serves as a foundation.

\section{A. Conceptual design}

Figure 3 provides a cross-section view and a conceptual illustration of the measuring segment of the force and distance sensing cannulation needle. A hollow shaft with four parallel grooves (three of which are effectively used) acts as a flexure for two opposing FBG fibres. These FBG fibres provide strain measurements due to bending in the yz-plane. These opposing strain measurements are used to determine the radial force component in the yz-plane exerted on the grooved shaft, while compensating for the thermal wavelength shift of the FBG fibres. A bent needle tip with known geometry is aligned to coincide with the y-direction. It is assumed that the puncture force predominantly acts along the axis of the needle tip. Therefore, knowing the geometric relationship of the needle tip to the measuring segment, a fair estimate of the puncture force can be derived. The puncture force estimate can then be used to determine the puncture force derivative. The force derivative signal, when combined with a previously developed puncture detection algorithm, 


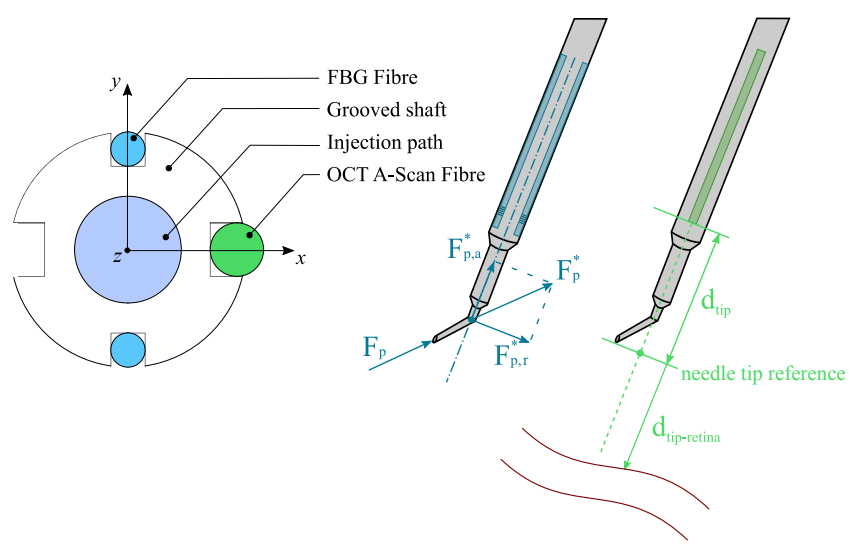

Fig. 3. Left: Cross-section of the sensor-integrated grooved shaft. Right: force sensing and distance sensing functionalities displayed separately.

has shown to provide a $98 \%$ success rate in detecting vessel punctures [5]. An OCT A-scan fibre is positioned in one of the remaining grooves, located on the neutral bending plane for loads in the y-direction. This configuration leads to a minimal increase of bending stifness of the instrument, and hence minimally affects the force sensor performance. The OCT A-scan fibre is oriented at the retinal surface. Unlike the design of the OCT A-scan fibre integrated retinal pick reported on by Iordachita et al. [13], no instrument feature is present within the OCT A-scan measurement window. Therefore, a calibration step is required in order to determine the needle tip reference.

\section{B. Detailed design}

Figure 4 provides an overview of the manufactured force and distance sensing cannulation needle. The outer handle is a stainless steel hollow shaft with threaded ends, which houses an internal assembly referred to as the injection track. At the distal end of the instrument, the injection track incorporates a Luer lock connector which is fixated to thinwalled stainless steel hollow shaft, which is present inside the entire length of the outer handle. At the proximal end of the first hollow shaft, a second stainless steel hollow shaft with 4 parallel outer grooves alongside its length, referred to as the grooved shaft, is inserted. The grooved shaft has an outer and inner diameter of respectively $550 \mu \mathrm{m}$ and 250 $\mu \mathrm{m}$, and two opposing pairs of grooves with widths of 100 $\mu \mathrm{m}$. Finally, at the proximal end of the grooved shaft a bent stainless steel microneedle assembly with a tip outer diameter of $80 \mu \mathrm{m}$ is inserted. Figure 5 provides a close-up of the used microneedle and the completed measuring segment. All stainless steel tubes are fixated using UV-curable medical adhesive. Fibres are aligned manually using a combination of precision linear stages, offering 3-DOF placement of the fibre with respect to the grooved shaft. Custom FBG fibers with an outer diameter of $80 \mu \mathrm{m}$ were used (FBGS Technologies $\mathrm{GmbH}$ ) in combination with an interrogator (SM130-500, Micron Optics). A custom made long range optical distance sensing fibre providing OCT A-scan data,

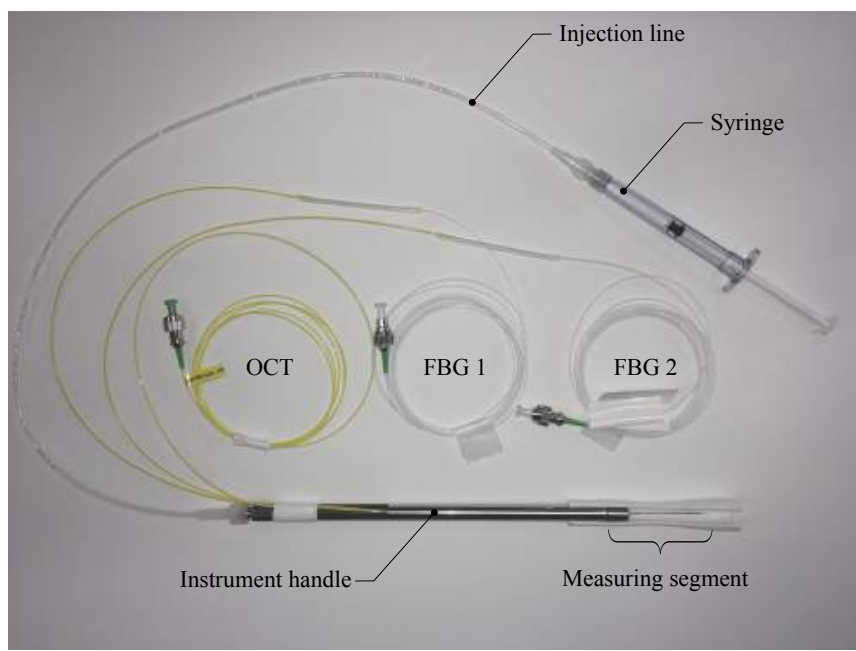

Fig. 4. The manufactured force and distance sensing cannulation instrument

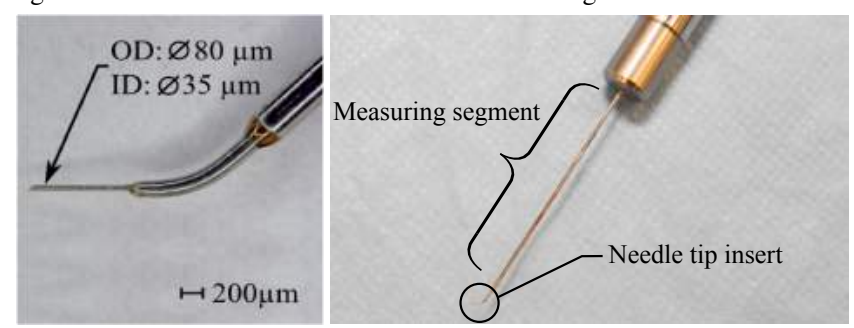

Fig. 5. Left: A close-up of the needle tip insert [12]. Right: A close up of the measuring segment of the manufactured force and distance sensing cannulation instrument.

with an outer diameter of $125 \mu \mathrm{m}$ was used (Medical Laser Center Lübeck GmbH). The OCT A-scan fibre is connected via an optical switch to an available medical grade iOCT scanner (OptoMedical Technologies $\mathrm{GmbH}$ ) mounted on a stereoscopic surgical microscope (Hi-R NEO 900A NIR, Haag-Streit Surgical GmbH). The two FBG fibres are aligned on opposite sides of the neutral bending axis of the tube. The FBG segments are placed at approximately $5 \mathrm{~mm}$ from the needle tip, therefore ensuring measured forces are only due to interaction forces of the instrument with the internal anatomy of the eye. The OCT A-scan fibre is aligned in one of the two remaining grooves, with the fibre focal point placed at approximately $1 \mathrm{~mm}$ distally from the needle tip reference. Once aligned, UV-curable medical adhesive is used to fixate the fibres. During the assembly process, a stereoscopic microscope with a magnification of up to $25 \mathrm{x}$ is used to visualise the work field.

\section{CALIBRATION AND CHARACTERISATION}

\section{A. Force data acquisition}

The available interrogator offers a wavelength detection resolution and a scan frequency of respectively $2 \mathrm{pm}$ and $500 \mathrm{~Hz}$. The wavelength-to-strain sensitivity of the used FBG fibers is $1 \mathrm{pm} / \mu \mathrm{m}$. A moving average filter with a window size of 50 samples is used, resulting in a response time of $0.1 \mathrm{~s}$. The measured strain in each fibre can be expressed as 
(1)

$$
\Delta \lambda=k_{\varepsilon} \varepsilon+k_{T} \Delta T
$$

\section{B. Force calibration}

The instrument handle is fixated horizontally, and oriented with the needle tip upwards. A calibration mass of $2 \mathrm{~g}$ is applied to the proximal end of the instrument. Once the calibration mass is stabilized (pendulum-like behaviour), 1000 wavelength samples are recorded and averaged for each fiber. Based on the linear relationship between the wavelength shift of both fibres and the applied radial force in the y-direction, a calibration factor $k_{f}$ is determined (2). Linear behaviour of the sensor across the force measurement range is assumed based on previous characterization of a similar design [12].

$$
\Delta \lambda_{1}-\Delta \lambda_{2}=k_{f} F_{y}
$$

\section{Force Characterisation}

Force measurement stability and precision is characterised both at zero and at maximum load condition. Based on the linear wavelength-to-force relationship and the symmetry of the sensor design in the measurement direction, a representative characterization for the entire measurement range is assumed. The instrument handle is fixated horizontally, and oriented with the needle tip upwards. A calibration mass of $2 \mathrm{~g}$ is applied to the proximal end of the instrument. Once the calibration mass is stabilized, measured force values are recorded for a duration of $45 \mathrm{~min}$. The same measurement is repeated without application of an external load. Figure 6(a) and Figure 6(b) show respectively the measured forces and the residual force error in function of time for both cases. No noticable drift is present for the zero loading condition, and minimal drift can be observed in the maximum load case, of up to $0.3 \mathrm{mN}$ over a timespan of 45 minutes. It is concluded that drift is negligible for the intended use of the instrument. The histogram depicted in Figure 6(c) shows that the residual errors are the largest for the maximum load case, with the 5th and 95th percentile points respectively being $0.10 \mathrm{mN}$ and $0.09 \mathrm{mN}$, indicating that 90 percent of the samples remain within a range of $0.19 \mathrm{mN}$ of the applied reference.

\section{Distance data acquisition}

OCT A-scan data is collected at a sample rate of 200 $\mathrm{Hz}$, at a pixel resolution of $3.8 \mu \mathrm{m}$. As a first proof of concept, a simple real-time distance sensing algorithm was implemented. Firstly, each A-scan vector is passed through a median filter for noise reduction. Secondly, a binary threshold conversion is performed on the filtered intensity values. Finally, a distance measurement is extracted by means of first feature recognition.

\section{E. Distance calibration}

The instrument handle is fixated horizontally, and aligned with a manually actuated precision translation stage with a resolution of $1 \mu \mathrm{m}$ (M-423 \& SM-25, Newport). The stage is translated in the direction of the OCT A-scan probe until first contact is observed with the aid of the microscope. Once

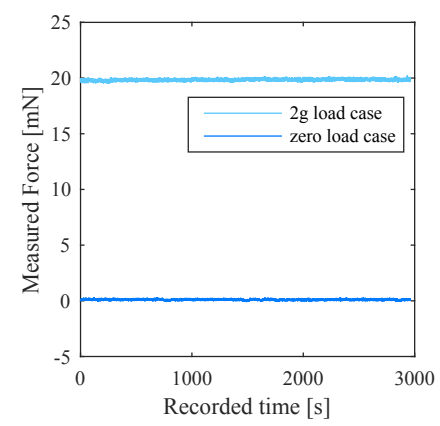

(a)

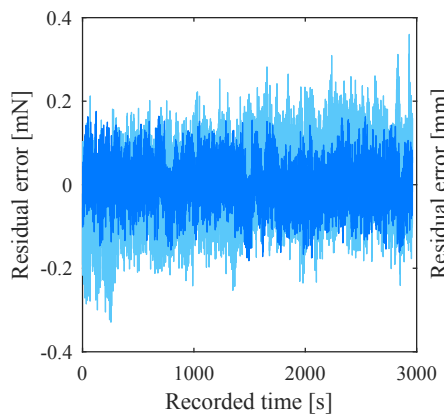

(b)

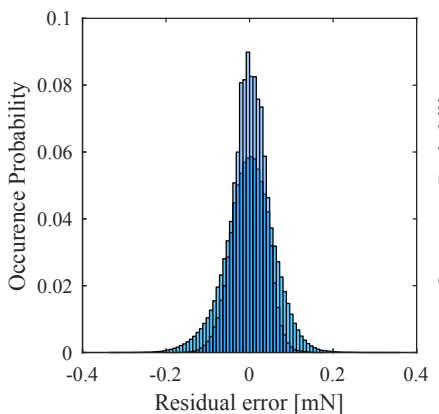

(c)

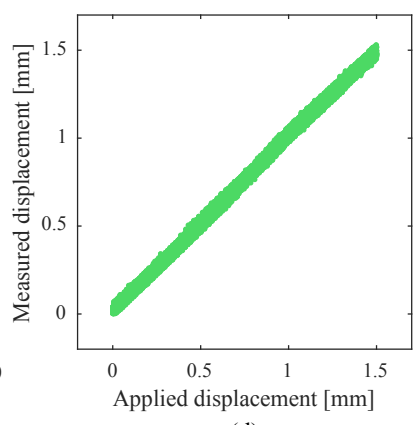

(d)

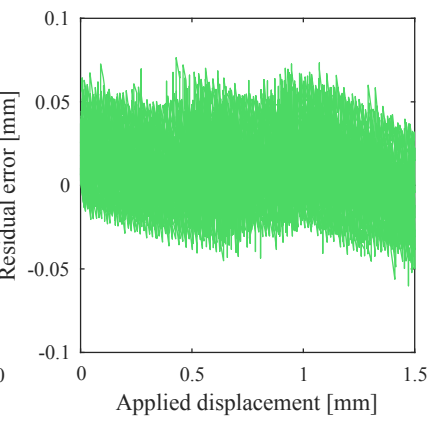

(e)

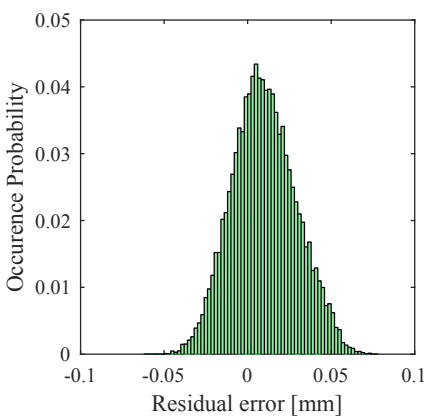

(f)
Fig. 6. (a) Measured forces, (b) force residual errors, and (c) histogram of the force residual errors for a $45 \mathrm{~min}$ registration period. (d) Measured displacement, (e) displacement residual error, and (f) histogram of the displacement residual error for a cyclic applied displacement of $1.5 \mathrm{~mm}$

the needle tip reference surface is set, 1000 A-scan samples are recorded and averaged. The distance reference retrieved from this measurement correlates the needle tip reference to the distance range of the OCT measurement window.

\section{F. Distance Characterisation}

Distance measurement precision and accuracy was characterized within the measurement window of $0 \mathrm{~mm}$ to $1.5 \mathrm{~mm}$, corresponding to a measurement range of $1.5 \mathrm{~mm}$ ahead of the needle tip. The instrument handle is fixated vertically to a precision single axis spindle drive (LX 26 series, Misumi) in combination with a position controlled DC-motor and encoder combination (RE30, Maxon Motors). This spindle and motor combination provides a positional resolution of $1 \mu \mathrm{m}$. A sinusoidal cyclic loading pattern with a period of $30 \mathrm{~s}$ providing a translation interval between 0 and $1.5 \mathrm{~mm}$ is applied for a duration of 10 minutes. Translational speed is limited to $500 \mu \mathrm{m} / \mathrm{s}$. Figure 6(d) shows the relationship 

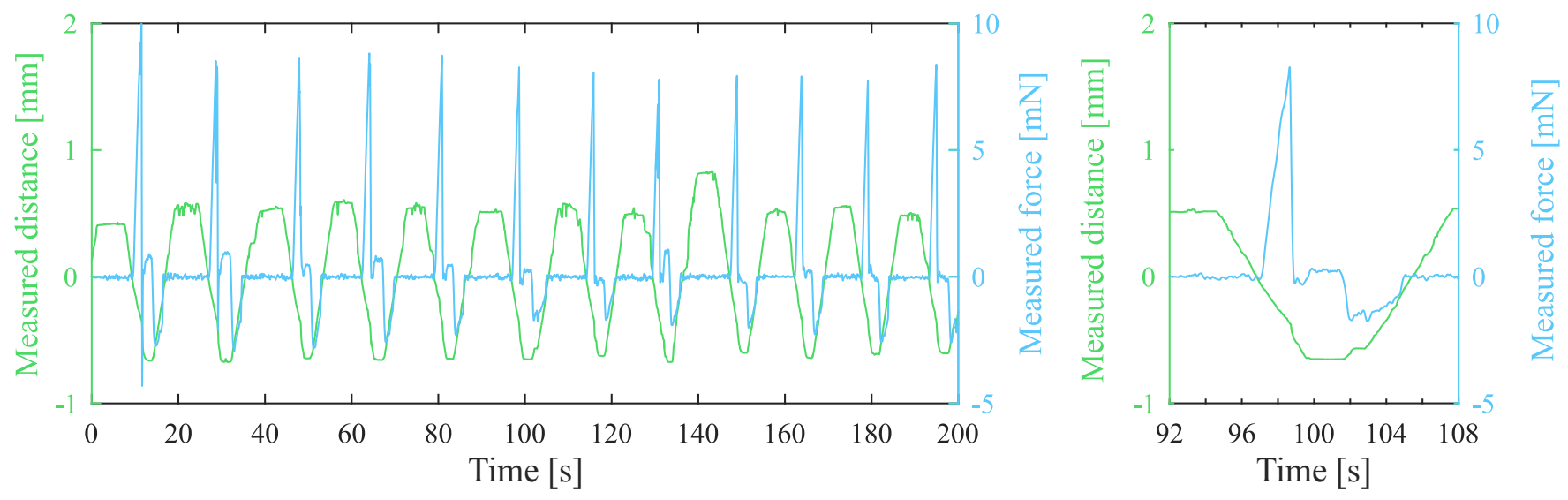

Fig. 7. Left: Measured distance and force values for a series of 12 punctures on a suspended membrane using a speed-controlled (0.5 mm/s) remotely operated test setup. Right: Isolated view of a typical puncture sequence.

between the applied and calculated displacement during the application of a cyclic load as described above. It is shown that the sensor in combination with the implemented distance sensing algorithm offers high repeatability and minimal hysteresis within the operated range. Figure 6(e) shows the residual error between the applied and calculated displacement in function of the applied displacement. Minor nonlinear behaviour can be observed. The histogram depicted in Figure 6(f) shows that the 5th and 95th percentile points are respectively $-0.021 \mathrm{~mm}$ and $0.043 \mathrm{~mm}$, indicating that 90 percent of the samples remain within a range of $0.064 \mathrm{~mm}$ of the applied reference. Mean error shows a deviation of $0.010 \mathrm{~mm}$ from the applied reference across the measurement range.

\section{EXPERIMENTAL VALIDATION}

\section{A. In-vitro robot-assisted needle punctures}

As a preliminary test, one user performed a series of 12 punctures on a suspended polyethylene foil with a thickness of $20 \mu \mathrm{m}$. This was found to produce comparable puncture force profiles to previous ex-vivo results performed using the same needle tip combined with a force sensitive fixture. For the purpose of this experiment, a dedicated test setup (Fig. 8 was developed to move the sensorized instrument in 1DOF. The instrument handle is fixated to a precision single axis spindle drive (LX 20 series, Misumi) in combination with a position controlled DC-motor and encoder combination (EC30, Maxon Motors). The needle tip axis is aligned with the translational orientation of the actuator. The user is able to control the instrument motion direction (up/down) with the aid of two foot pedals, at a fixed translational speed of $500 \mu \mathrm{m} / \mathrm{s}$. With the aid of a manually actuated rotative and translational precision stage (M-423, M-481, \& SM25 , Newport), the needle tip height and angle of attack relative to the target surface are set. Figure 7 depicts the measured force and distance values in function of time. Each puncture sequence can be categorized in four periods. Firstly, the user approaches the target. The distance between the target surface and the needle tip gradually decreases

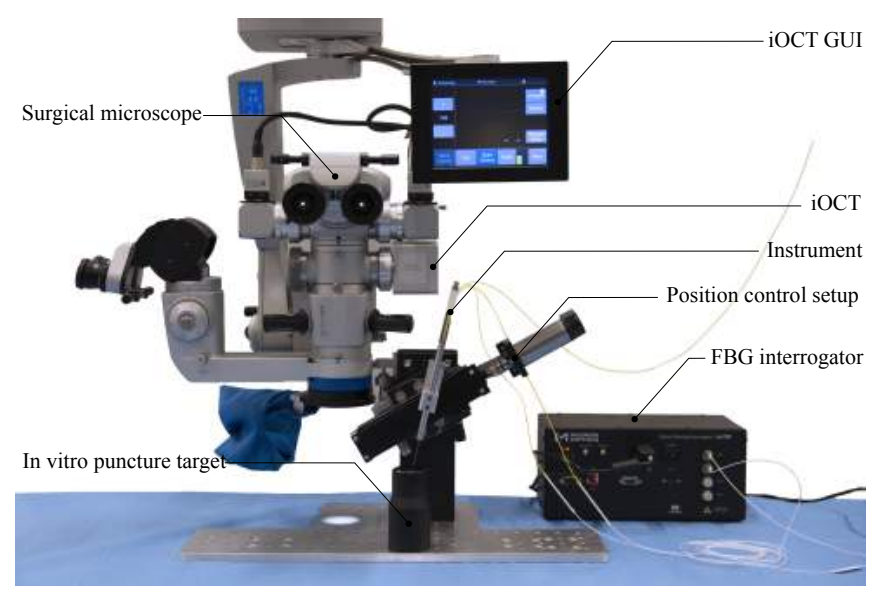

Fig. 8. Overview of the used materials to perform in-vitro cannulations.

while forces remain around zero. Secondly, a steep positive rise in force level followed by a negative drop in force level indicates respectively needle tip contact and puncture. At this time, the user stops the instrument motion causing the measured distance value to remain constant. Thirdly, a negative force value paired with a rise in measured distance indicates the start of needle retraction, followed by the needle tip being retracted from the target once the force returns to zero. Finally, the user returns to the initial position at approximately $0.5 \mathrm{~mm}$ above the target. At this point, the cycle is repeated.

\section{B. Ex-vivo distance estimation}

The instrument handle is fixated vertically to a precision single axis spindle drive (LX 20 series, Misumi) in combination with a position controlled DC-motor and encoder combination (EC30, Maxon Motors). This spindle and motor combination provides a positional resolution of $0.25 \mu \mathrm{m}$. A sinusoidal cyclic loading pattern with a period of $30 \mathrm{~s}$ providing a translation interval between 0 and $1.5 \mathrm{~mm}$ was applied for a duration of 10 minutes. Translational speed was limited to $500 \mu \mathrm{m} / \mathrm{s}$. Four ex-vivo enucleated porcine eyes 


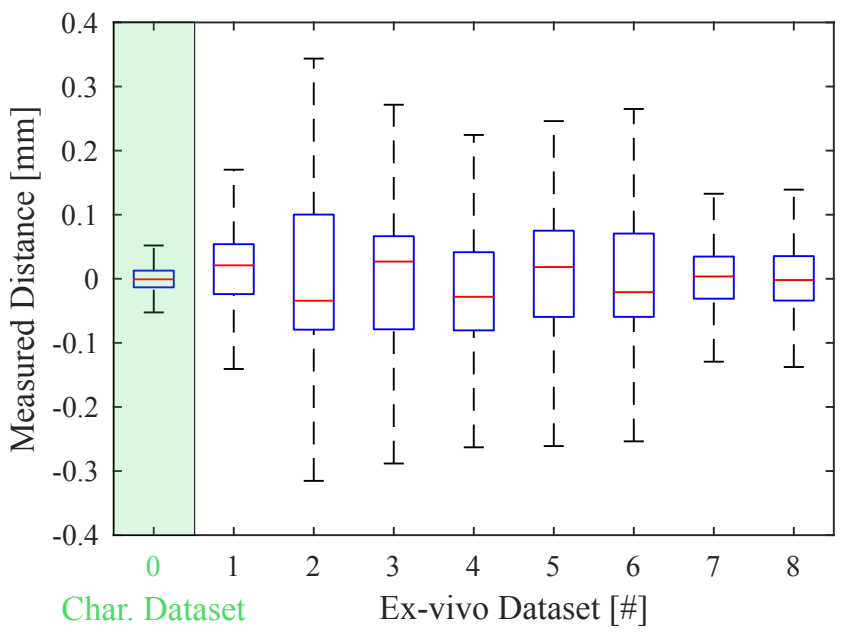

Fig. 9. Boxplots of the mean-normalized residual distance error for each ex-vivo dataset. Boxes indicate the 25-75 percentile range, limits indicate full range with outliers removed. The calibration dataset of Figure 6 (e) is shown for relative comparison

were used in two different orientations, providing 8 datasets. Figure 9 shows the outcomes of the ex-vivo experimental validation of the distance estimation algorithm. For each dataset, a boxplot of the residual error between the applied and calculated displacement is shown. The characterisation dataset is shown for relative comparison. The mean precision (5-95 percentile range) across all ex-vivo datasets is determined to be $0.239 \mathrm{~mm}$, as opposed to the $0.064 \mathrm{~mm}$ precision achieved during characterisation. It is concluded that the implemented algorithm suffers a factor 3.7 drop in precision when implemented ex vivo.

\section{DISCUSSION}

This work reported on the development of a novel combined force and distance sensing cannulation needle for retinal vein cannulation. The force and distance measurements rely respectively on 2 FBG fibres and a single OCT A-scan fibre which were integrated into a $0.55 \mathrm{~mm}$ instrument shaft. Characterisation of the force-sensing-modalities showed a force precision of $0.2 \mathrm{mN}$ for a maximum response time of $0.1 \mathrm{~s}$. Characterisation of the distance-sensing-modalities showed a distance accuracy and precision of respectively $0.010 \mathrm{~mm}$ and $0.064 \mathrm{~mm}$ for a maximum response time of $0.1 \mathrm{~s}$. Based on in-vitro experimental validation, it was shown that the developed sensorized instrument is able to reliably display puncture events, as well as display a stable realtime estimate of the distance of the needle tip to the target surface. Ex-vivo experimental validation of the implemented distance sensing algorithm shows that while distance estimation remains feasible, precision drops a factor 3.7 relative to reference surface characterisation. It is hypothesized that this is mainly due to the reduced reflectance of the $e x$ vivo tissue. These ex-vivo results highlight the need for further development of real-time distance estimation algorithms in order to enable ex-vivo and in-vivo usage. Future work includes the development of more advanced distance estimation algorithms, and the integration of the developed sensorized instrument with a surgical robotic system.

\section{ACKNOWLEDGEMENTS}

This research was funded by The EU Framework Programme for Research and Innovation - Horizon 2020 - Grant Agreement No 645331 and an SB Fellowship of the Research Foundation - Flanders (1S41517N).

\section{REFERENCES}

[1] S. Rogers, R. L. Mcintosh, B. Grad, D. Journ, N. Cheung, L. Lim, J. J. Wang, P. Mitchell, J. W. Kowalski, H. Nguyen, and T. Y. Wong, "The Prevalence of Retinal Vein Occlusion: Pooled Data from Population Studies from the United States, Europe, Asia, and Australia," Population Studies, vol. 117, no. 2, pp. 1-14, 2011.

[2] W. M. Tang and D. P. Han, "A Study of Surgical Approaches to Retinal Vascular Occlusions," Archives of Ophthalmology, vol. 118, no. 1, pp. 138-143, jan 2000.

[3] J. N. Weiss, "Treatment of central retinal vein occlusion by injection of tissue plasminogen activator into a retinal vein," American Journal of Ophthalmology, vol. 126, no. 1, pp. 142-144, 1998.

[4] T. Donald, "Robot Assists With Injections For RVO," Retina Today, no. April, pp. 59-61, apr 2017.

[5] A. Gijbels, K. Willekens, L. Esteveny, P. Stalmans, D. Reynaerts, and E. B. Vander Poorten, "Towards a clinically applicable robotic assistance system for retinal vein cannulation," in 6th IEEE International Conference on Biomedical Robotics and Biomechatronics (BioRob), 2016, pp. 284-291.

[6] A. Molaei, E. Abedloo, M. D. de Smet, S. Safi, M. Khorshidifar, H. Ahmadieh, M. A. Khosravi, and N. Daftarian, "Toward the Art of Robotic-assisted Vitreoretinal Surgery," Journal of Ophthalmic \& Vision Research, vol. 12, no. 2, pp. 175-182, 2017.

[7] "KU Leuven Eye Surgery Robot." [Online]. Available: www.eyesurgery-robot.be

[8] A. D. Jagtap and C. N. Riviere, "Applied force during vitreoretinal microsurgery with handheld instruments," in The 26th Annual International Conference of the IEEE Engineering in Medicine and Biology Society, vol. 1, 2004, pp. 2771-2773.

[9] X. He, J. Handa, P. Gehlbach, R. Taylor, and I. Iordachita, "A Submillimetric 3-DOF Force Sensing Instrument With Integrated Fiber Bragg Grating for Retinal Microsurgery," IEEE Transactions on Biomedical Engineering, vol. 61, no. 2, pp. 522-534, 2014.

[10] B. Gonenc and I. Iordachita, "FBG-based transverse and axial force-sensing micro-forceps for retinal microsurgery," in 2016 IEEE SENSORS, oct 2016, pp. 1-3.

[11] B. Gonenc, R. H. Taylor, I. Iordachita, P. Gehlbach, and J. Handa, "Force-sensing microneedle for assisted retinal vein cannulation," in IEEE SENSORS 2014 Proceedings, 2014, pp. 698-701.

[12] A. Gijbels, E. B. Vander Poorten, P. Stalmans, and D. Reynaerts, "Development and Experimental Validation of a Force Sensing Needle for Robotically Assisted Retinal Vein Cannulations," in 2015 IEEE International Conference on Robotics and Automation (ICRA), 2015, pp. 2270-2276.

[13] M. Balicki, J.-H. Han, I. Iordachita, P. Gehlbach, J. Handa, R. Taylor, and J. Kang, "Single Fiber Optical Coherence Tomography Microsurgical Instruments for Computer and Robot-Assisted Retinal Surgery," in Medical Image Computing and Computer-Assisted Intervention - MICCAI 2009. Berlin, Heidelberg: Springer, 2009, pp. 108-115.

[14] G. Borghesan, D. Bouget, E. Lankenau, R. Neffin, and P. Koch, "Single Fiber OCT-based Retina Detection for Robot-assisted Retinal Vein Cannulation," in Proceedings of the 6th Joint Workshop on New Technologies for Computer/Robot Assisted Surgery, 2016.

[15] G. W. Cheon, Y. Huang, J. Cha, P. L. Gehlbach, and J. U. Kang, "Accurate real-time depth control for CP-SSOCT distal sensor based handheld microsurgery tools." Biomedical Optics Express, vol. 6, no. 5, pp. 1942-1953, 2015.

[16] J. Smits, M. Ourak, A. Gijbels, G. Borghesan, L. Esteveny, L. Schoevaerdts, K. Willekens, P. Stalmans, E. Lankenau, G. Hüttmann, D. Reynaerts, and E. B. V. Poorten, "Combined Force and Distance Sensing for Robot-Assisted Vitreo-Retinal Surgery," in Proceedings of the 7th Joint Workshop on New Technologies for Computer/Robot Assisted Surgery, 2017. 